\title{
A Corresponding States Model for Generalized Engineering Equations of State
}

\author{
L. Sun ${ }^{1,2}$ and J. F. Ely ${ }^{1,3}$
}

Received July 30, 2004

\begin{abstract}
In this work, a four-parameter corresponding-states principle (CSP) model is proposed to generalize the universal technical Equation of State (EOS) developed in our previous work. This model is in the form of the Helmholtz free energy and takes the reduced density, reduced temperature, acentric factor and a polarity factor as variables. Compared to other generalized equations such as the one by Span and Wagner for nonpolar fluids, and by Platzer and Maurer and by Wilding and Rowley for polar fluids, the CSP model developed in this work shows good accuracy for the 22 nonpolar, polar, and associating fluids considered in this study and offers the flexibility to be extended to other fluids of industrial interest. In addition, the polarity factor used in this model has been successfully correlated from quantitative structure activity relationship (QSAR) molecular descriptors.
\end{abstract}

KEY WORDS: corresponding states principle; equation of state; polar and nonpolar fluids; thermodynamic properties.

\section{INTRODUCTION}

Efforts to develop equations of state (EOS) as sources of accurate thermodynamic property prediction have been the focus of research for more than a century. Ever since the pioneering work of van der Waals in 1873, there have been many different types of EOS proposed. They include the modified cubic [1-3], virial [4] and its derivatives like the BWR-type EOS [5], and, for example, the molecular-based statistical associating fluids theory

\footnotetext{
${ }^{1}$ Chemical Engineering Department, Colorado School of Mines, Golden, Colorado 80401, U.S.A.

${ }^{2}$ Present Address: Chemical Engineering Department, Vanderbilt University, Nashville, Tennessee 37235, U.S.A.

${ }^{3}$ To whom correspondence should be addressed. E-mail: jely@mines.edu
} 
(SAFT) EOS [6, 7]. The BWR-type EOS proposed by Benedict et al. [8], is an early example of the so-called multiparameter equation of state, which is solely based on regression of experimental data, and whose accuracy is definitely superior to a cubic equation. Newer versions, like the modified BWR-type equation (the MBWR-32, proposed by Jacobsen and Stewart [9]), were regarded as the first examples of reference EOS which are able to describe the experimental data to within their experimental uncertainties over a wide range of state conditions and have good extrapolation behavior in the regions not covered by experimental data. Nonetheless, the successful development of accurate EOS essentially lies on the quality of the experimental data available.

Since high quality experimental data and highly accurate reference equations are available for relatively few fluids such as water [10], carbon dioxide [11], nitrogen [12], argon [13], etc., moderate quality technical EOS are good choices for fluids with a varying quality of data. However, for the majority of industrially interesting fluids, insufficient experimental data are available so that even a technical EOS can be developed. These fluids either possess small data sets covering limited thermodynamic states, or existing data show large inconsistencies.

In order to overcome this problem, accurate predictive approaches are needed to estimate the thermodynamic properties for such fluids from a knowledge of existing well-represented fluids. The corresponding-states principle is widely used in engineering applications as an effective tool for generalizing equations and making these predictions. A generalized technical EOS is expected to describe a broad range of fluids which are relevant to the chemical and petrochemical industries with reasonable accuracy and numerical stability. Moreover, generalized technical EOS are considered to be highly valuable when handling fluid mixtures.

For simple molecules or conformal fluids, the corresponding states principle (CSP) is a two-parameter theory for which the intermolecular potential is only related to distance and not by the relative orientation of the two molecules. For more complex molecules, additional parameters need to be introduced to construct more complicated corresponding states principles. According to Pitzer [14], the acentric factor $\omega$ is the most useful and readily available parameter. Lee and Kesler extended Pitzer's method to calculate properties in the critical and low-temperature regions [15]. In their method, a reference fluid, in addition to the simple fluid, was introduced so that properties of other fluids can be calculated by interpolation and extrapolation. Wilding and Rowley [16] further extended the LK model (ELK) to polar fluids by introducing a polarity factor $\beta$ and replacing the acentric factor $\omega$ with a shape/size factor $\alpha$. Water was introduced 
as the second reference fluid, while methane and $n$-octane were kept as the simple fluid and the first reference fluid.

In addition to the CSP, there are several other ways to generalize equations, such as the semi-empirical models like the BACKONE equation of state [17], shape factor models [18], and group contribution methods [19]. Cubic equations of state are not listed, since they are not flexible enough to reach a good balance of simplicity and accuracy. A large body of research has been performed to assess the performance of the abovementioned approaches in a more or less systematic way [20-25]. The results of these comparisons show that in most cases these generalized equations of state cannot meet the requirements for advanced technical equations of state. Part of the problem lies in the quality of substance specific equations of state, which constitute the basis for correlations. For example, the quality of the modified BWR EOS that was used in the LeeKesler model is not high enough to meet the needs for high accuracy engineering applications. Thus, research to find other ways to generalize a technical equation of state remains active.

Platzer and Maurer [26] proposed a four-parameter approach to generalize the coefficients in the Bender-type EOS. The approach by Platzer and Maurer [26] is completely predictive, since there is no fit involved and the approach only requires four substance-related parameters. However, the results obtained from this approach do not satisfy the requirements of accuracy and numerical stability for a generalized technical EOS, as pointed out by Span and Wagner [27]. Span and Wagner [27] used one additional parameter $w$ instead of the acentric factor $\omega$ and the polarity factor $\beta$ to correlate the coefficients of their technical EOS. Their proposed generalized EOS is in the form of reduced Helmholtz free energy with 30 coefficients, and the structure of the base equation was optimized by using the SIMOPT algorithm [28].

Strictly speaking, neither Platzer and Maurer's nor Span and Wagner's approach are rigorous corresponding states approaches. As described in Section 2, the corresponding states principle, in practice, is extended by additional parameters following a truncated Taylor expansion. The truncated Taylor expansion keeps only the first-order derivatives with respect to the additional parameters, and higher-order derivatives are neglected. However, in Platzer and Maurer's approach, there is one cross term and one power term of the acentric factor and polarity factor. The inclusion of these terms is not justified by the authors. Similarly in Span and Wagner's approach, there is a 4th power term present in the correlation which is not justified in a corresponding states sense.

In this work, we consider the universal technical equation developed in our previous work [29] as the base equation and use propane, $n$-octane, 
and water as three reference fluids in the corresponding states approach proposed by Wilding and Rowley [16]. Instead of obtaining values for the polarity parameter from experimental data, we propose a method to determine these values from molecular descriptors.

We shall proceed as follows. In Section 2, we consider the theoretical background for the corresponding states principle. In Section 3, we describe a method to establish the corresponding states model based on a wide-range multiparameter equation of state. The new four-parameter model and its comparison with other models are given in Section 4. Our conclusions are summarized in Section 5.

\section{CORRESPONDING STATES PRINCIPLE}

There are two types of corresponding states theories, the classical theory and the molecular theory of corresponding states. These two theories are actually related to each other, and the difference lies in the reducing parameters. The molecular theory of corresponding states is based on mathematical properties of the potential-energy function [30]. For spherical molecules, the dimensionless potential will be a universal function of the reduced dependent variable, i.e., the dimensionless distance between molecules. Since the macroscopic thermodynamic properties of a specified substance can be calculated by the techniques of statistical mechanics given the potential function, a universal equation of state (EOS) can be logically obtained from a known universal potential function. The classical theory of corresponding states proposed by van der Waals is based on the mathematical properties of his macroscopic EOS. According to this theory, an EOS is a universal function of the reduced coordinates, expressed as

$$
\frac{P}{P_{c}}=F\left(\frac{T}{T_{c}}, \frac{V}{V_{c}}\right)
$$

where $P$ is pressure, $T$ is temperature, $V$ is volume, and the subscript $c$ refers to the critical point. The connection between the parameters of molecular theory of corresponding states and the parameters of the classical theory are obtained from the critical-point criteria. At the critical point, there are only two independent variables chosen from $P, V$, and $T$ since the compressibility at the critical point $z_{c}$ is the same for all fluids in a two-parameter equation of state. In engineering application, the corresponding states principle is usually expressed in the form of the compressibility factor,

$$
z=z\left(T_{r}, P_{r}\right)
$$


where $T_{r}=T / T_{c}$ and $P_{r}=P / P_{c}$. In this work we focus on the application of the classical corresponding states principle. For more details about the molecular theory of corresponding states, the readers should refer to references by Prausnitz et al. [30] and by Ely and Marrucho [31].

For more complex molecules, additional parameters need to be introduced to construct a corresponding states model. According to Pitzer [14], the acentric factor is introduced as a measure of the non-central nature of intermolecular forces, and can be calculated from experimental data from its definition,

$$
\omega \equiv-\log \left(\frac{P_{s}}{P_{c}}\right)_{T / T_{c}=0.7}-1,
$$

where $P_{s}$ is the saturation pressure. The three-parameter corresponding states principle then becomes

$$
z\left(T_{r}, P_{r}, \omega\right)=z_{0}\left(T_{r}, P_{r}, \omega=0\right)+\omega z_{1}\left(T_{r}, P_{r}\right),
$$

where $z_{0}$ is the compressibility of a simple fluid for which $\omega=0$ and $z_{1}$ is the deviation of a real fluid from $z_{0}$.

In Lee and Kesler's extension of Pitzer's method [19], a reference fluid in addition to the simple fluid was introduced, so that properties of other fluids can be calculated by interpolation and extrapolation. The compressibility factor $z$ of a specified fluid is then written in the form,

$$
z\left(T_{r}, P_{r}, \omega\right)=z_{0}\left(T_{r}, P_{r}, \omega=0\right)+\frac{\omega}{\omega_{1}}\left[z_{1}\left(T_{r}, P_{r}, \omega_{1}\right)-z_{0}\left(T_{r}, P_{r}, \omega=0\right)\right],
$$

where the subscript 1 refers to the reference fluid, and $z_{0}$ and $z_{1}$ can be calculated from a modified BWR equation. Lee and Kesler chose methane as the simple fluid and $n$-octane as the reference fluid. Teja et al. [32, 33] have extended the LK model, so that any two non-spherical fluids can be used as reference fluids. Their results are written as follows:

$$
z=z_{r 1}+\frac{\omega-\omega_{r 1}}{\omega_{r 2}-\omega_{r 1}}\left[z_{r 2}-z_{r 1}\right]
$$

where subscript $r_{1}$ refers to the first reference fluid and $r_{2}$ to the second. The Lee and Kesler (LK) model represents nonpolar fluids very well, and can be applied to some weakly polar fluids. However, it cannot be applied to strongly polar fluids.

Wilding and Rowley [16] have extended the LK model (ELK) to polar fluids by introducing a polarity factor $\beta$ and replacing the acentric factor 
$\omega$ with a shape/size factor $\alpha$. Water was introduced as the second reference fluid while methane and $n$-octane were kept as the simple fluid and the first reference fluid. The ELK model is given by

$$
z\left(T_{r}, P_{r}\right)=z_{0}\left(T_{r}, P_{r}\right)+\alpha z^{(1)}\left(T_{r}, P_{r}\right)+\beta z^{(2)}\left(T_{r}, P_{r}\right),
$$

where

$$
z^{(1)}=\frac{\left(z_{1}-z_{0}\right)}{\alpha_{1}}
$$

and

$$
z^{(2)}=\left[\left(z_{2}-z_{0}\right)-\frac{\alpha_{2}}{\alpha_{1}}\left(z_{1}-z_{0}\right)\right] .
$$

A modified BWR equation was used to calculate the compressibility factor for methane and $n$-octane. However, for water, the EOS developed by Keenan et al. [34] was used since the modified BWR does not give good predictions for water. Although the ELK model predicts fairly good results for a wide range of polar fluids, efforts to create corresponding states models are continuing.

\section{AN APPROACH TO GENERALIZE THE TECHNICAL EOS}

We propose a new approach to develop a generalized EOS based on previous research $[15,16,32,33]$ on the corresponding states principle and on our newly developed technical EOS [29]. Several features of this approach are given in the following:

(i) The approach will use two additional parameters, the acentric factor $\omega$ and a polarity factor $\beta$, in addition to the critical temperature $T_{c}$ and the critical density $\rho_{c}$.

(ii) A predictive correlation for the polarity factor $\beta$ will be determined from the technical equations of state and sets of molecular descriptors for a given substance.

(iii) The approach will be established based on a truncated Taylor expansion, retaining only the first-order derivatives with respect to the two additional parameters.

(iv) The approach will use two non-spherical fluids, propane and $n$-octane, as the references for nonpolar fluids, and water as the reference for polar fluids.

(v) The approach will be based on the technical EOS developed in previous work [1], in the form of the reduced Helmholtz free energy. 
Following the procedures proposed by Wilding and Rowley [16], any dimensionless configurational property $J$ of a specified fluid at a given reduced temperature $T_{r}=T / T_{c}$ and density $\rho_{r}=\rho / \rho_{c}$, may be represented as

$$
J\left(T_{r}, \rho_{r}\right)=J_{0}\left(T_{r}, \rho_{r}\right)+\left(\omega-\omega_{0}\right) J^{(1)}\left(T_{r}, \rho_{r}\right)+\left(\beta-\beta_{0}\right) J^{(2)}\left(T_{r}, \rho_{r}\right),
$$

where

$$
J^{(1)}=\frac{\left(J_{1}-J_{0}\right)}{\omega_{1}-\omega_{0}}
$$

and

$$
J^{(2)}=\frac{1}{\beta_{2}-\beta_{0}}\left[\left(J_{2}-J_{0}\right)-\frac{\left(\omega_{2}-\omega_{0}\right)}{\left(\omega_{1}-\omega_{0}\right)}\left(J_{1}-J_{0}\right)\right] .
$$

$J_{0}$ is the corresponding property of the first non-spherical, nonpolar reference fluid with $\omega_{0} \neq 0$ and $\beta_{0}=0, J_{1}$ is the property of the second nonspherical, nonpolar reference fluid with $\omega_{1} \neq 0$ and $\beta_{1}=0$, and $J_{2}$ is the property of a non-spherical and polar reference fluid with $\omega_{2} \neq 0$ and $\beta_{2}=$ 1. Note that even though $\beta_{2}-\beta_{0}=1$ in our case, we formally retained the term in the expression to better present the idea of calculating properties from three reference fluids.

The technical EOS developed is expressed in the form of the dimensionless Helmholtz free energy as shown in Ref. 29;

$$
\begin{gathered}
\Phi \equiv \frac{A(\rho, T)}{R T}=\Phi^{\mathrm{id}}(\delta, t)+\Phi^{r}(\delta, t), \\
\Phi^{r}(\delta, t)=\sum_{m=1}^{M_{1}} a_{m} \delta^{i_{m}} t^{j_{m}}+\sum_{m=M_{1}+1}^{M_{2}} a_{m} \delta^{i_{m}} t^{j_{m}} \exp \left(-\delta^{k_{m}}\right),
\end{gathered}
$$

where $\Phi$ is the dimensionless Helmholtz free energy and the reduced variables are $\delta=\rho / \rho_{c}=\rho_{r}$ and $t=T_{c} / T=1 / T_{r}$. The ideal gas contributions were calculated with the functional form and parameters given in Ref. 27. As shown in Eq. (14), the residual part of the dimensionless Helmholtz free energy $\Phi^{r}(\delta, t)$ is a universal function of the two reduced properties $\delta$ and $t$. If we replace $J$ in Eq. (10) with $\Phi^{r}(\delta, t)$, we obtain a four-parameter corresponding states model in the form of the residual dimensionless Helmholtz energy written as

$$
\Phi^{r}(\delta, t)=\Phi_{0}^{r}(\delta, t)+\left(\omega-\omega_{0}\right) \Phi^{(1)}(\delta, t)+\left(\beta-\beta_{0}\right) \Phi^{(2)}(\delta, t)
$$


with

$$
\Phi^{(1)}=\frac{\left(\Phi_{1}^{r}-\Phi_{0}^{r}\right)}{\omega_{1}-\omega_{0}}
$$

and

$$
\Phi^{(2)}=\frac{1}{\beta_{2}-\beta_{0}}\left[\left(\Phi_{2}^{r}-\Phi_{0}^{r}\right)-\frac{\left(\omega_{2}-\omega_{0}\right)}{\left(\omega_{1}-\omega_{0}\right)}\left(\Phi_{1}^{r}-\Phi_{0}^{r}\right)\right],
$$

where subscripts 0,1 , and 2 refer to the first and second non-spherical, nonpolar reference fluid and a third non-spherical, polar reference fluid, respectively. Even though the polarity factor $\beta$ is not experimentally measurable, it can in principle be solved for by nonlinearly fitting Eq. (15) to the thermophysical properties of a given fluid, or as shown below, determined directly from the equation of state for a given fluid.

In our approach the equations of state used to calculate properties for the three reference fluids have the same functional form as shown in Eq. (14) but with different sets of coefficients $a_{m}$. That simplifies the corresponding states model (Eq. (15)) by correlating the coefficients $a_{m}$ directly with the four parameters. Assuming the polynomial terms $\delta^{i_{m}} t^{j_{m}}$ and exponential terms $\delta^{i_{m}} t^{j_{m}} \exp \left(-\delta^{k_{m}}\right)$ are unique at the same reduced properties $\delta$ and $t$, we can write the corresponding states model (Eq. (15)) in a form where the coefficients $a_{m}$ are given by

$$
\begin{gathered}
a_{m}=a_{m, 0}+\left(\omega-\omega_{0}\right) a^{(1)}+\left(\beta-\beta_{0}\right) a^{(2)}, \\
a^{(1)}=\frac{\left(a_{m, 1}-a_{m, 0}\right)}{\omega_{1}-\omega_{0}},
\end{gathered}
$$

and

$$
a^{(2)}=\frac{1}{\beta_{2}-\beta_{0}}\left[\left(a_{m, 2}-a_{m, 0}\right)-\frac{\left(\omega_{2}-\omega_{0}\right)}{\left(\omega_{1}-\omega_{0}\right)}\left(a_{m, 1}-a_{m, 0}\right)\right] .
$$

The subscripts have the same definitions as in Eq. (15). Because of this simplification, the polarity parameter $\beta$ can be solved for directly from Eqs. (18-20) for a given fluid, without the need of fitting experimental data. The corresponding states model we propose gives a generalized technical EOS with the form shown in Eqs. (13) and (14). The coefficients $a_{m}$ for a specified fluid are determined through Eq. (18).

The determination of $\omega$ and $\beta$ relies on information for the critical parameters, and uncertainties in these parameters can lead to erroneous values of $\omega$ and $\beta$, especially for $\beta$. Therefore, since the polarity factor 
$\beta$ reflects microscopic information about a molecule, correlation models should relate the values of $\beta$ to certain types of molecular descriptors. Modern molecular simulation software like Cerius ${ }^{2}$ [35] provides powerful tools to model a molecule at a quantum mechanical and statistical mechanical level. The user can "build" a molecule and calculate the microscopic properties of that molecule which can then be used in a quantitative structure-activity (or property) relationship (QSAR/QSPR) technique. The QSAR module in Cerius ${ }^{2}$ software addresses questions as to what features of a molecule affect its activity and what can be modified to enhance properties. Usually a QSAR correlation is expressed as a linear mathematical model to account for the observed activity;

$$
Y=f\left(x_{1}, x_{2}, \ldots, x_{n}\right) .
$$

The success of a QSAR correlation depends on the accuracy of the dependent variable $Y$ and proper choice of the independent variables $x$. The independent variables are those physiochemical properties that describe some aspect of the chemical structure. They may be determined experimentally or calculated, and they are often referred to as descriptors. Typical molecular descriptors are spatial, electronic, thermodynamic, conformational, topological, information-content, quantum mechanical, and structural descriptors based on fragment constants, descriptors based on receptor surface models (RSA), descriptors based on molecular field analysis (MFA), and descriptors based on molecular shape analysis (MSA) [36].

A preliminary investigation of the correlation between the polarity factor $\beta$ of the fluids studied and all molecular descriptors in the QSAR module showed that a linear QSAR model is not accurate enough to meet the requirements for a generalized technical EOS. The values of $\beta$ calculated from such a linear correlation showed large deviations from the values determined by fitting the coefficients of the EOS as described above. Moreover, when linearly correlated values of $\beta$ are incorporated in Eq. (15), the resulting CSP model gives substantial deviations from experimental data. Therefore, we developed a method to correlate the polarity factor $\beta$ with molecular descriptors by using the stepwise regression method. The selected molecular descriptors used in this work are the spatial descriptors - molecular surface area $(A)$, volume $(V)$, radius of gyration $(R)$, and an electronic descriptor - the dipole moment $(D)$. These descriptors are combined in power forms and put in a bank of terms as given by

$$
\sum A^{i} V^{j} D^{k} R^{l}
$$


Here the exponents $i, j, k$, and $l$ are arbitrarily determined numbers, and values of the molecular descriptors are available for each fluid from the study table in the Cerius ${ }^{2}$ software. The bank of terms allows cross and power terms, which are not available in the QSAR module. Similar to the procedures used in developing a multiparameter equation of state from experimental data, the correlation model for $\beta$ is determined by using the step-wise regression algorithm and has the form,

$$
\beta=\sum_{n=1}^{N} b_{n} A^{i_{n}} V^{j_{n}} D^{k_{n}} R^{l_{n}} .
$$

\section{RESULTS AND DISCUSSION}

The generalized technical equation takes the forms in Eqs. (13) and (14), and the coefficients $a_{m}$ for each fluid are calculated from Eq. (18) together with Eqs. (19) and (20). In these equations, subscripts 0, 1, and 2 refer to propane and $n$-octane as the non-spherical, nonpolar reference fluids and water as the non-spherical, polar reference fluid, respectively. The equation structure and corresponding coefficients $a_{m, i}(i=0,2)$ from previous work [29] are given in Tables I and II. Table III presents the critical constants, acentric factors, and the polarity factors $\beta$ used in this work. The correlation model developed for $\beta$ has the form of Eq. (23)

Table I. Exponents of the Simultaneously Optimized Technical Equation of State

\begin{tabular}{rlll}
\hline$m$ & $i_{m}$ & $j_{m}$ & $k_{m}$ \\
\hline 1 & 1 & 1.50 & 0 \\
2 & 1 & 0.25 & 0 \\
3 & 1 & 1.25 & 0 \\
4 & 3 & 0.25 & 0 \\
5 & 7 & 0.875 & 0 \\
6 & 2 & 1.375 & 0 \\
7 & 1 & 0.00 & 1 \\
8 & 1 & 2.375 & 1 \\
9 & 2 & 2.00 & 1 \\
10 & 5 & 2.125 & 1 \\
11 & 1 & 3.50 & 2 \\
12 & 1 & 6.50 & 2 \\
13 & 4 & 4.75 & 2 \\
14 & 2 & 12.50 & 3 \\
\hline
\end{tabular}


Table II. Coefficients of the Simultaneously Optimized Equation of State for the Reference Fluids

\begin{tabular}{rrrr}
\hline & \multicolumn{3}{c}{$a_{m}$} \\
\cline { 2 - 4 }$m$ & Propane & Octane & Water \\
\hline \multirow{2}{*}{1} & $9.70439249 \times 10^{-1}$ & $1.57750154 \times 10^{0}$ & $3.46821920 \times 10^{-1}$ \\
2 & $9.73671323 \times 10^{-1}$ & $1.15745614 \times 10^{0}$ & $5.03423025 \times 10^{-1}$ \\
3 & $-2.96661981 \times 10^{0}$ & $-3.54867092 \times 10^{0}$ & $-3.51059570 \times 10^{-1}$ \\
4 & $7.84340496 \times 10^{-2}$ & $1.18030671 \times 10^{-1}$ & $5.07004866 \times 10^{-2}$ \\
5 & $2.78440866 \times 10^{-4}$ & $3.02753897 \times 10^{-4}$ & $1.99939129 \times 10^{-4}$ \\
6 & $-6.77622221 \times 10^{-2}$ & $-2.63074957 \times 10^{-1}$ & $-5.69888763 \times 10^{-1}$ \\
7 & $-8.56371936 \times 10^{-2}$ & $2.55299486 \times 10^{-2}$ & $-1.96198912 \times 10^{-1}$ \\
8 & $1.77467443 \times 10^{-1}$ & $-1.26632996 \times 10^{-1}$ & $-2.02509554 \times 10^{0}$ \\
9 & $3.91636018 \times 10^{-1}$ & $4.48343319 \times 10^{-1}$ & $-1.09353609 \times 10^{0}$ \\
10 & $-8.03312946 \times 10^{-3}$ & $-9.46702997 \times 10^{-3}$ & $7.25785202 \times 10^{-2}$ \\
11 & $-2.60385851 \times 10^{-1}$ & $-4.43927529 \times 10^{-1}$ & $2.16072642 \times 10^{-1}$ \\
12 & $-1.91104746 \times 10^{-2}$ & $-1.68224827 \times 10^{-2}$ & $-1.01542630 \times 10^{-1}$ \\
13 & $-6.31331470 \times 10^{-2}$ & $-1.15864640 \times 10^{-1}$ & $7.46926106 \times 10^{-2}$ \\
14 & $-2.27769095 \times 10^{-2}$ & $-1.32417591 \times 10^{-2}$ & $2.18830463 \times 10^{-3}$ \\
\hline & & &
\end{tabular}

with $N=11$. The coefficients $a_{m}, b_{n}$, and exponents $i, j, k$, and $l$ in this model are given in Tables IV. Overall, the correlation model gives very good agreement with the fitted values, i.e., the average absolute deviation is 0.008 , the average deviation (BIAS) is -0.001 , and the root-mean-square deviation (RMS) is 0.012. Comparisons between the CSP model and the generalized equation of Span and Wagner (SWG) [27] for nonpolar and weakly polar fluids are given in Section 4.1. Section 4.2 presents the comparisons between the CSP model and the models by Platzer and Maurer [26] and Wilding and Rowley [16] for polar and associating fluids.

\subsection{Nonpolar and Weakly Polar Fluids}

The generalized equation proposed by Span and Wagner [27] exhibits better accuracy for the nonpolar and weakly polar fluids as compared to the generalized model by Platzer and Maurer [26]. Therefore, only the equation by Span and Wagner (SWG) is used for comparisons of the 13 fluids considered here. However, since Span and Wagner do not include benzene and toluene in their model, values calculated from the Platzer and Maurer equation are used for comparison with these two fluids.

Table V presents the overall comparison of selected experimental data between the CSP model and the SWG equation for the 13 nonpolar and 
Table III. Summary of Critical Parameters, Acentric Factors, and Polarity Factor for Fluids Studied in this Work

\begin{tabular}{lccccl}
\hline Substances & $\mathrm{T}_{\mathrm{c}}(\mathrm{K})$ & $\mathrm{P}_{\mathrm{c}}(\mathrm{MPa})$ & $\rho_{\mathrm{c}}\left(\mathrm{kg} \cdot \mathrm{m}^{-3}\right)$ & $\omega$ & $\beta$ \\
\hline Methane & 190.564 & 4.599 & 162.66 & 0.011 & 0 \\
Ethane & 305.330 & 4.872 & 206.60 & 0.099 & 0 \\
Ethylene & 282.35 & 5.042 & 214.24 & 0.087 & 0 \\
Propane & 369.825 & 4.248 & 220.48 & 0.153 & 0 \\
$n$-Butane & 407.817 & 3.640 & 224.36 & 0.185 & 0 \\
$i$-Butane & 425.125 & 3.796 & 227.84 & 0.200 & 0 \\
$n$-Pentane & 553.600 & 4.078 & 273.02 & 0.209 & 0 \\
$n$-Hexane & 469.700 & 3.370 & 232.00 & 0.251 & 0 \\
Cyclohexane & 507.820 & 3.034 & 233.18 & 0.308 & 0 \\
$n$-Octane & 569.320 & 2.497 & 234.90 & 0.391 & 0 \\
Benzene & 561.750 & 4.8758 & 304.63 & 0.211 & 0 \\
Toluene & 593.950 & 4.2365 & 290.24 & 0.264 & 0 \\
Nitrogen & 126.192 & 3.396 & 313.30 & 0.037 & 0 \\
Carbon Dioxide & 304.128 & 7.3773 & 467.60 & 0.225 & 0.0125 \\
R32 & 351.350 & 5.795 & 427.00 & 0.277 & 0.25 \\
R134a & 339.330 & 3.629 & 571.30 & 0.304 & 0.125 \\
R125 & 374.180 & 4.0563 & 508.00 & 0.327 & 0.0125 \\
Ammonia & 405.400 & 11.340 & 225.00 & 0.256 & 0.625 \\
Methanol & 512.600 & 8.104 & 269.14 & 0.566 & 0.5 \\
Ethanol & 513.920 & 6.132 & 276.00 & 0.637 & 0.4 \\
1-Propanol & 536.710 & 5.184 & 273.20 & 0.628 & 0.35 \\
Water & 647.096 & 22.064 & 322.00 & 0.348 & 1 \\
\hline
\end{tabular}

Table IV. Coefficients and Exponents of STW-QSAR Correlations for Polarity Factor $\beta$

\begin{tabular}{rrrllr}
\hline$n$ & $b_{n}$ & $i_{n}$ & $j_{n}$ & $k_{n}$ & $l_{n}$ \\
\hline 1 & $-0.235517301001 \times 10^{6}$ & -2 & -2 & 0 & 1 \\
2 & $0.625738254831 \times 10^{6}$ & -2 & -2 & 1 & -1 \\
3 & $-0.293826438876 \times 10^{7}$ & -2 & -2 & 1.5 & 2 \\
4 & $0.167125009406 \times 10^{8}$ & -2 & -2 & 2 & -2 \\
5 & $-0.814294420014 \times 10^{8}$ & -2 & -2 & 2 & -1 \\
6 & $0.150053430831 \times 10^{9}$ & -2 & -2 & 2 & 0 \\
7 & $-0.125589638489 \times 10^{9}$ & -2 & -2 & 2 & 1 \\
8 & $0.426366893003 \times 10^{8}$ & -2 & -2 & 2 & 2 \\
9 & $0.569963417448 \times 10^{5}$ & -2 & -1.5 & 0 & 0 \\
10 & $-0.139390654099 \times 10^{4}$ & -1 & -2 & 0 & -2 \\
11 & $-0.417163348224 \times 10^{-1}$ & -1 & 1.5 & 2 & -1 \\
\hline
\end{tabular}




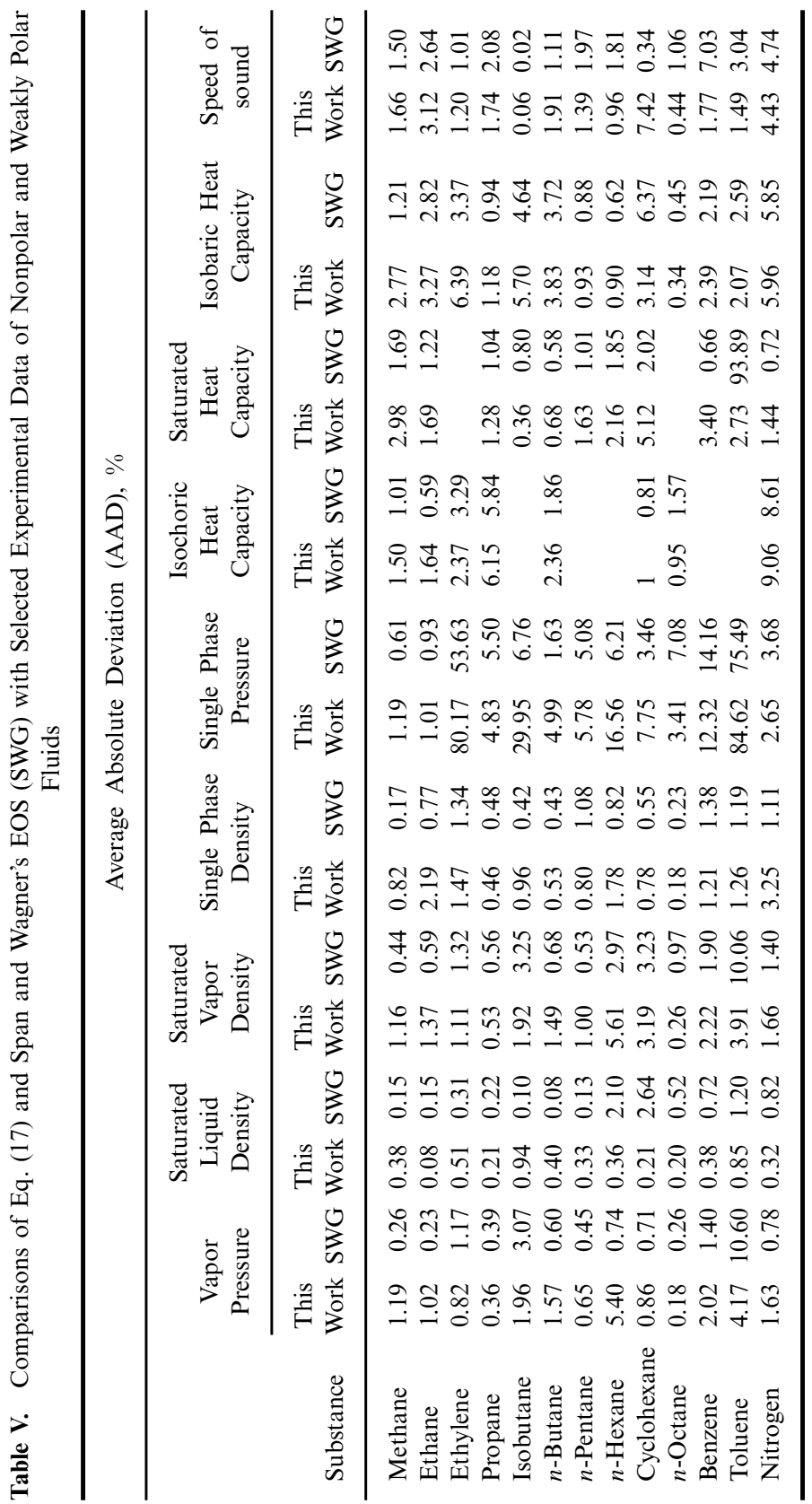




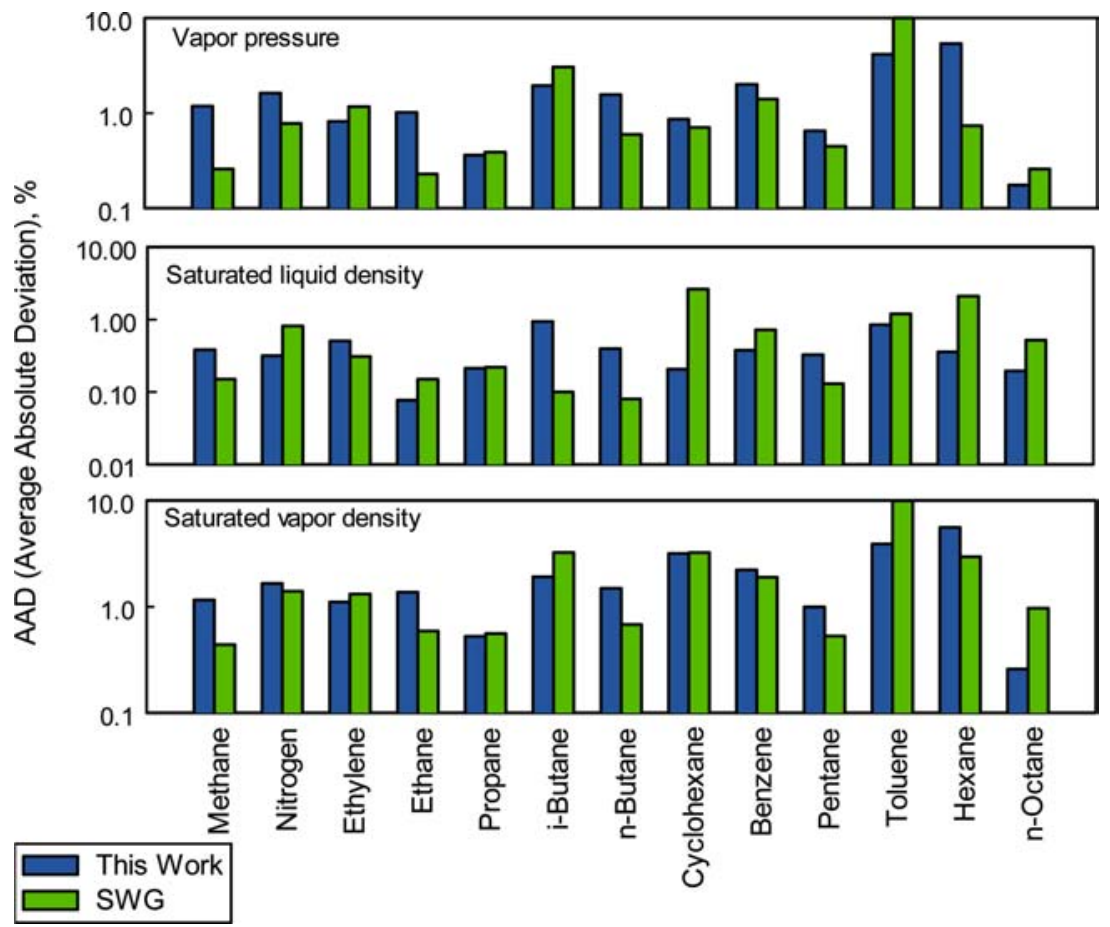

Fig. 1. Comparisons of average absolute deviations for saturation boundary calculated from the CSP model and the equations by Span and Wagner [27] for 13 nonpolar and weakly polar fluids.

weakly polar fluids. Clearly the CSP and SWG models give similar predictions for the entire range of fluids. Figure 1 presents the comparisons of the saturation boundary between the CSP model and the SWG equation. The CSP model predicts the saturation boundary properties within $1 \%$ for most of the fluids except for $i$-butane, toluene, and hexane, while the SWG equation gives larger deviations for $i$-butane, $n$-hexane, and $n$-octane. The CSP model also shows better accuracy for benzene and toluene when compared to the equation by Platzer and Maurer.

In Fig. 2, comparisons of the predictions for single-phase densities are shown. Again, the CSP model gives accurate predictions for all 13 fluids with deviations generally less than $2 \%$ except for nitrogen. The SWG gives better predictions for methane, nitrogen, ethane, $i$-butane, and $n$-hexane and the CSP model is better for $n$-pentane and $n$-octane. For the rest of fluids, the two models show similar accuracy. The equation by Platzer and Maurer gives similar predictions for benzene and toluene. 


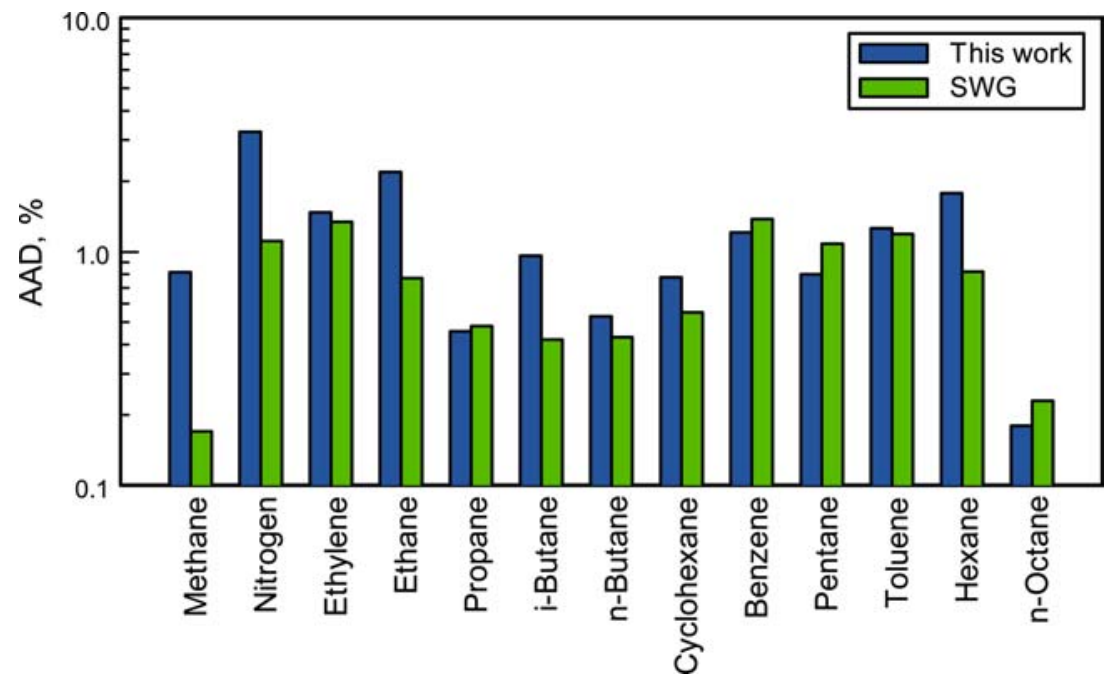

Fig. 2. Comparisons of average absolute deviations for single-phase densities calculated from the CSP model and the equations by Span and Wagner [27] for 13 nonpolar and weakly polar fluids.

Figure 3 shows comparisons of the isobaric heat capacity data. The SWG equation gives better predictions for methane, ethylene, and $n$-hexane, and the CSP model exhibits better accuracy for cyclohexane and $n$-octane. Their accuracies are about the same for the remaining fluids, including benzene (the results for benzene are very close) and toluene when compared to the equation by Platzer and Maurer [26]. Generally, however, the isobaric heat capacity data can only be predicted to within $7 \%$.

In Fig. 4, comparisons of speed-of-sound data between the CSP model and the SWG equation are shown. The SWG equation gives good predictions for $i$-butane and cyclohexane, while the CSP model shows better predictions for $n$-pentane, $n$-hexane, $n$-octane, benzene, and toluene. For all fluids except cyclohexane, the speed-of-sound data can be predicted within $4 \%$ by the CSP model.

Clearly the generalized equation proposed in this work based on the corresponding-states principle (CSP) possesses similar quality in predicting thermodynamic properties when compared to the generalized equation by Span and Wagner for all 13 nonpolar and weakly polar fluids considered. We should note here, however, that two adjustable parameters in addition to $w$ are required in order to achieve the high accuracy in the SWG model. These two parameters, namely, $T_{\text {ref }}$ and $\rho_{\text {ref }}$, are different values 


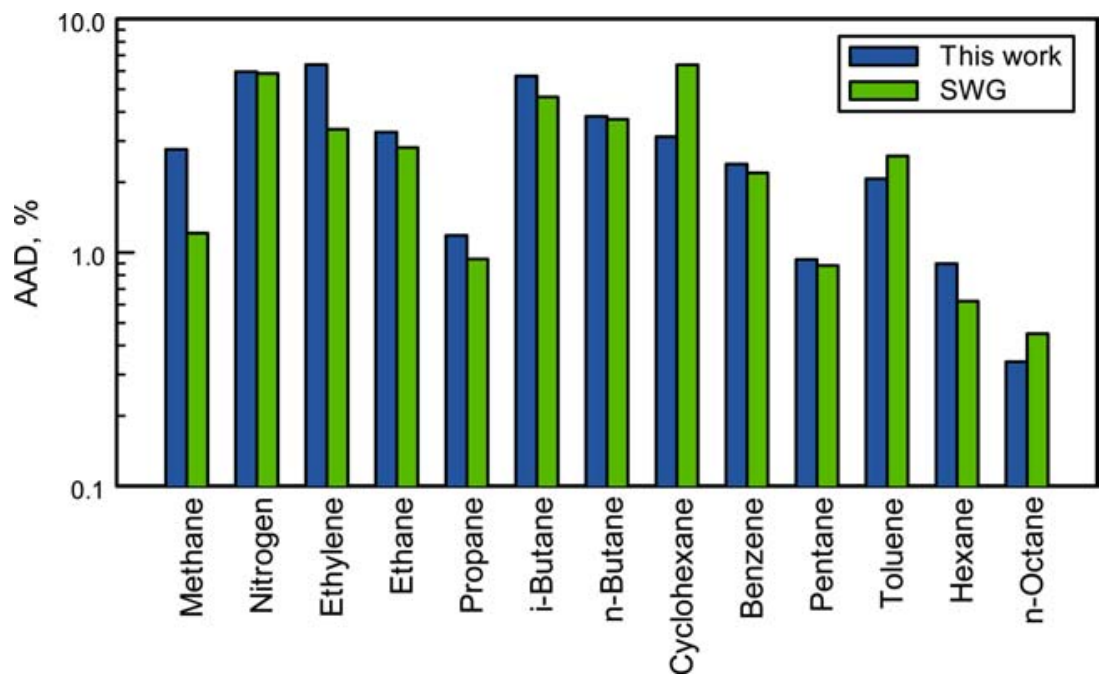

Fig. 3. Comparisons of average absolute deviations for isobaric heat capacities calculated from the CSP model and the equations by Span and Wagner [27] for 13 nonpolar and weakly polar fluids.

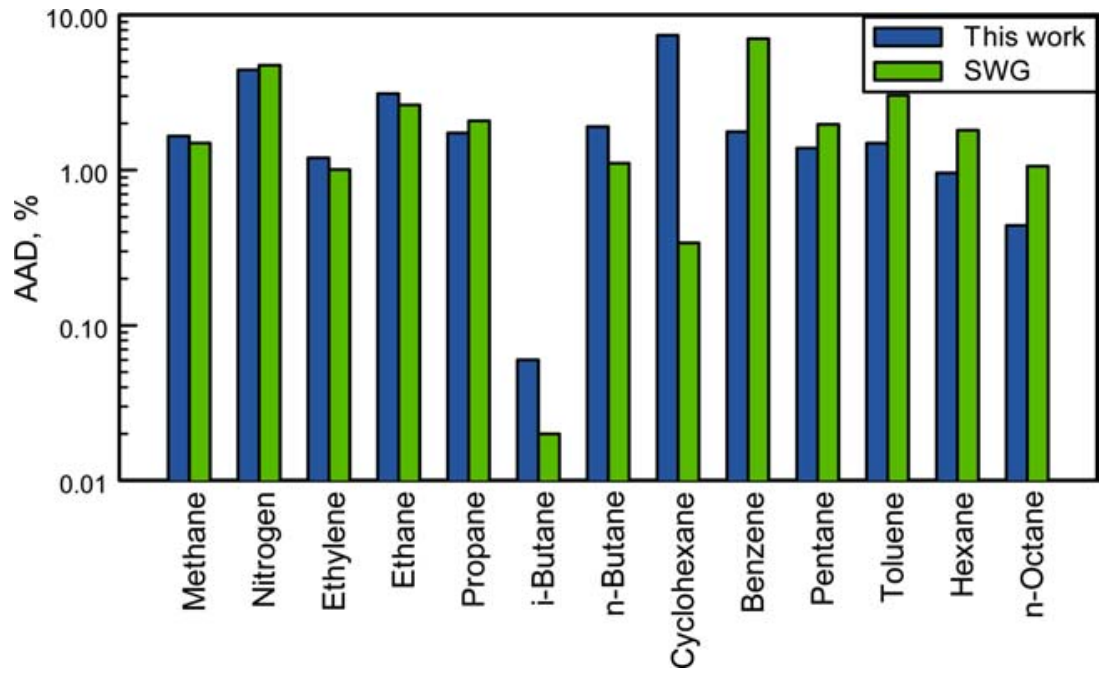

Fig. 4. Comparisons of average absolute deviations for speed of sound data calculated from the CSP model and the equations by Span and Wagner [27] for 13 nonpolar and weakly polar fluids. 


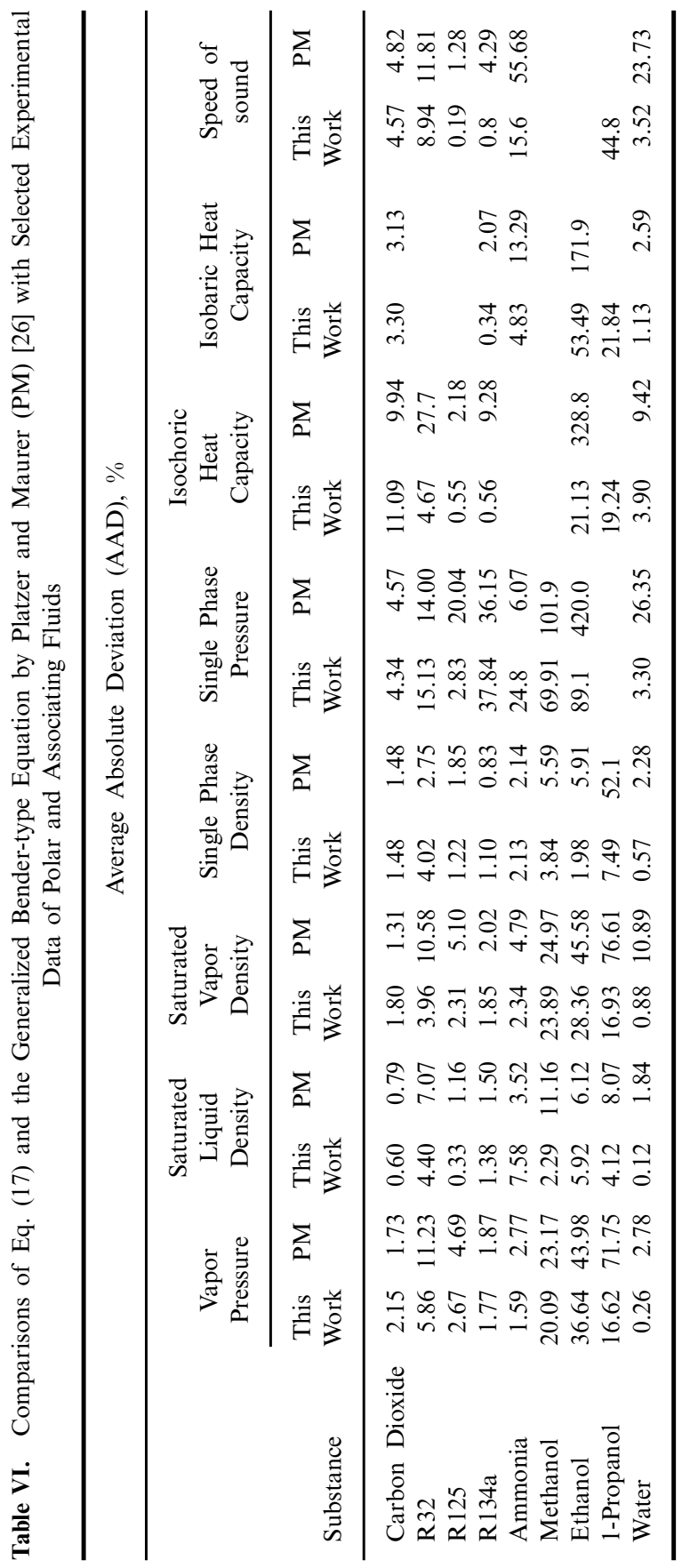


from the critical parameters $T_{c}$ and $\rho_{c}$ and have to be fitted for each individual fluid from experimental data. Therefore, although the SWG model offers a generalized functional form, the fitting of substance specific parameters limits the predictive power of the SWG model to those fluids with abundant experimental data. More importantly, the generalized equation by Span and Wagner [2] is restricted to nonpolar and weakly polar fluids and is not applicable to polar fluids.

\subsection{Polar and Associating Fluids}

The generalized Bender-type equation proposed by Platzer and Maurer (PM) [26], and the generalized MBWR equation by Wilding and Rowley (WR) [16] are used in this part of our work for comparisons of nine polar and associating fluids. Platzer and Maurer [26] did not publish the values of the polarity factor $\chi$ for R32, R134a, R125, ethanol, and 1propanol; therefore, the values are predicted in this work following their method that uses the vapor pressure. Similarly, Wilding and Rowley [16] did not give values for the size/shape factor $\alpha$ for R32, R134a, R125, and carbon dioxide, and their values are predicted using their correlation equation for $\alpha$. Table VI presents the overall comparisons for selected experimental data between the CSP model and the generalized Bendertype equation by Platzer and Maurer [26]. Clearly the CSP model gives better predictions over the PM equation for the entire range of fluids.

Figure 5 shows the comparisons of the saturation-boundary properties between the CSP model and the PM equation. Since water is used as a reference fluid in the CSP model, the comparison for water is irrelevant. For other fluids, the CSP model predicts better results for vapor pressure and the saturated vapor density. The exceptions are carbon dioxide and ammonia. For carbon dioxide, slightly worse results are seen for the vapor pressure and the saturated vapor density. As for ammonia, worse results are obtained with the CSP model. Comparing to the predictions on the saturation boundary for nonpolar fluids, the results for polar fluids are close except for the alcohols.

Figure 6 shows the comparisons of the saturated liquid densities again, but this time comparisons with the generalized equation of Wilding and Rowley are also included. The WR equation gives larger deviations for the polar fluids in general, but better predictions for 1-propanol and ethanol over the CSP and the PM equations. Also, the WR equation shows a better quality of predictions for the alcohols and water over the PM equation.

Figure 7 represents the comparisons of single-phase densities. The CSP model gives slightly better or similar predictions over the PM equa- 


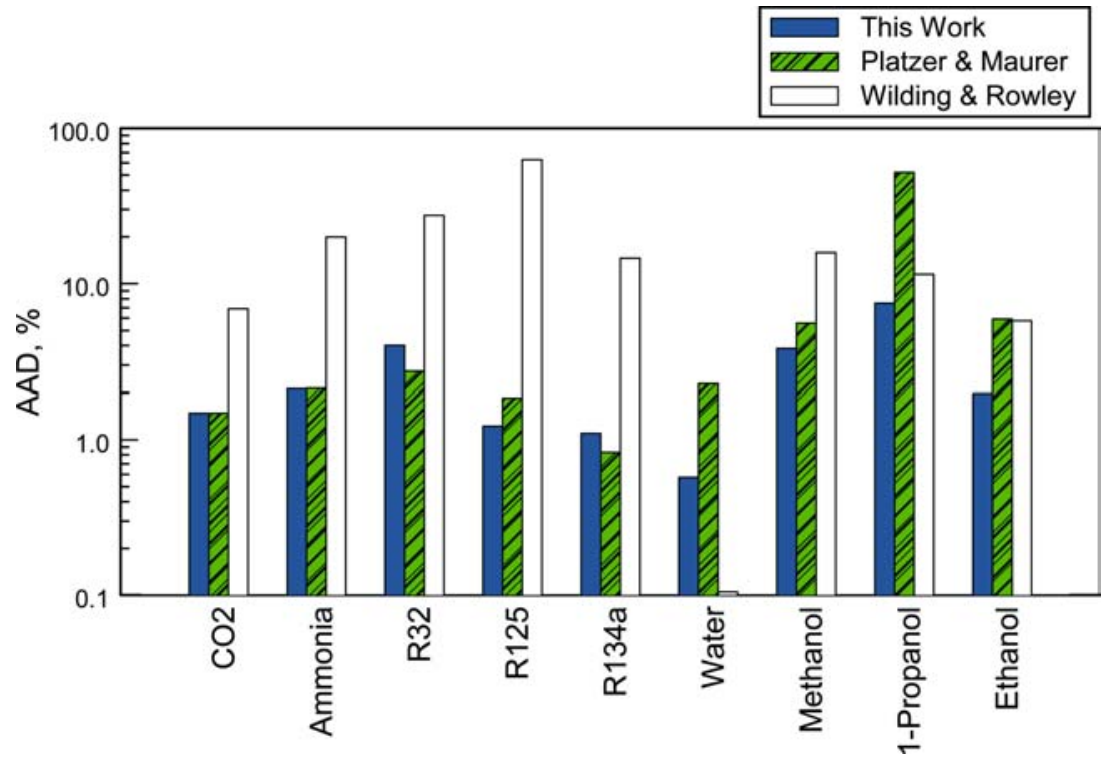

Fig. 5. Comparisons of average absolute deviations for single densities calculated from the CSP model and the equations by Platzer and Maurer [26] and by Wilding and Rowley [16] for 9 polar and associating fluids.
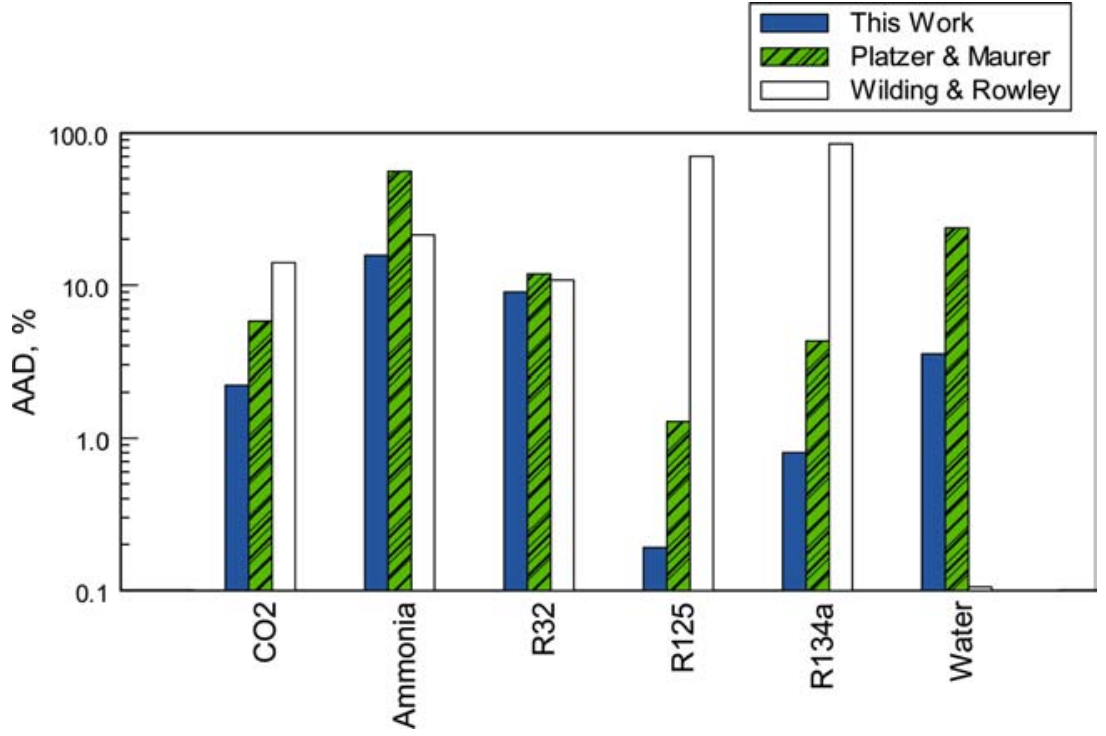

Fig. 6. Comparisons of average absolute deviations for speed of sound data calculated from the CSP model and the equations by Platzer and Maurer [26] and by Wilding and Rowley [16] for 9 polar and associating fluids. 


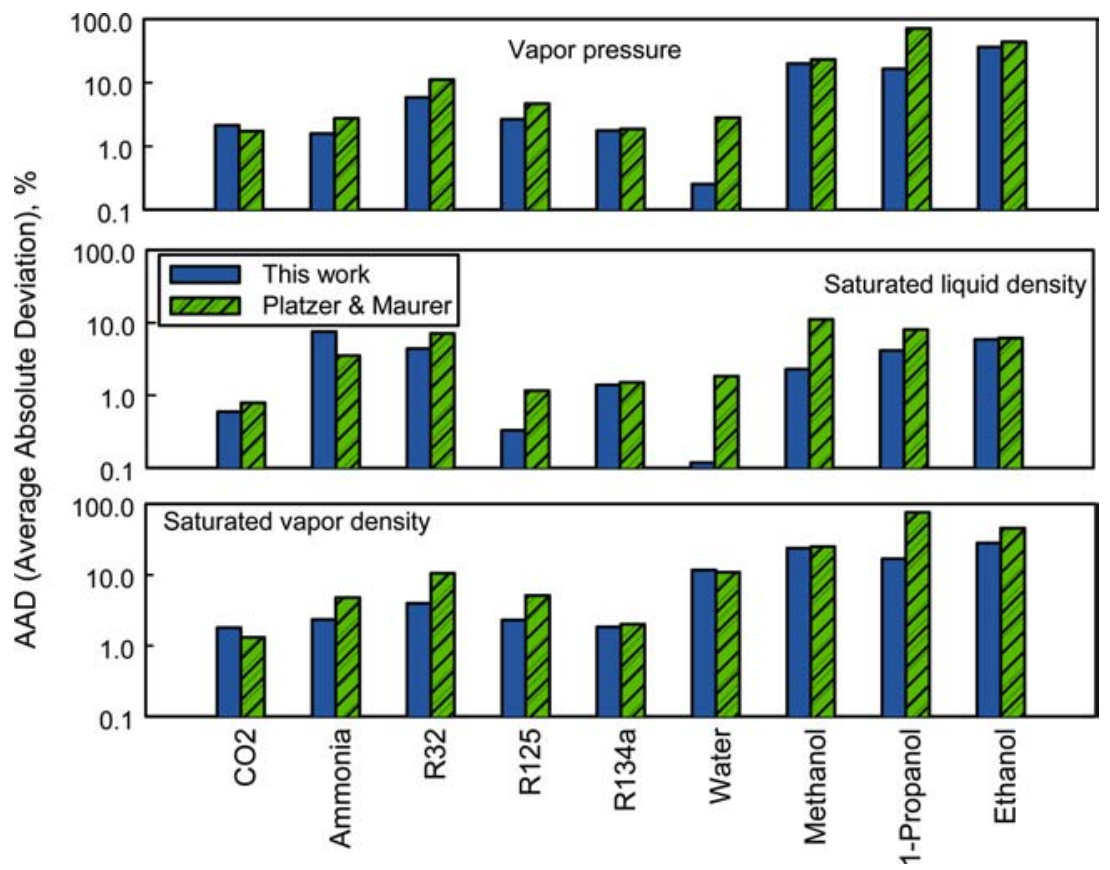

Fig. 7. Comparisons of average absolute deviations for saturation boundary calculated from the CSP model and the equation by Platzer and Maurer [26] for 9 polar and associating fluids.

tion for carbon dioxide, ammonia, and R125. For R32 and R134a the PM is slightly better. However, for the associating fluids, the CSP model is much better as compared to the PM and WR equations. A reference quality equation for water is used in Wilding and Rowley's approach; therefore, the predictions for water are meaningless. The WR equation gives the largest deviations for polar fluids among the three models compared.

Figure 8 compares the speed-of-sound data. Since all three models do not give accurate predictions for the alcohols, results for methanol, ethanol, and 1-propanol are not included in this figure although the CSP model gives smaller deviations. For the polar fluids considered here, the CSP model gives better predictions than the PM and WR equations. All three models give very close results for R32. The deviations calculated from the CSP are less than 10\% except for ammonia, which exceed $15 \%$. 


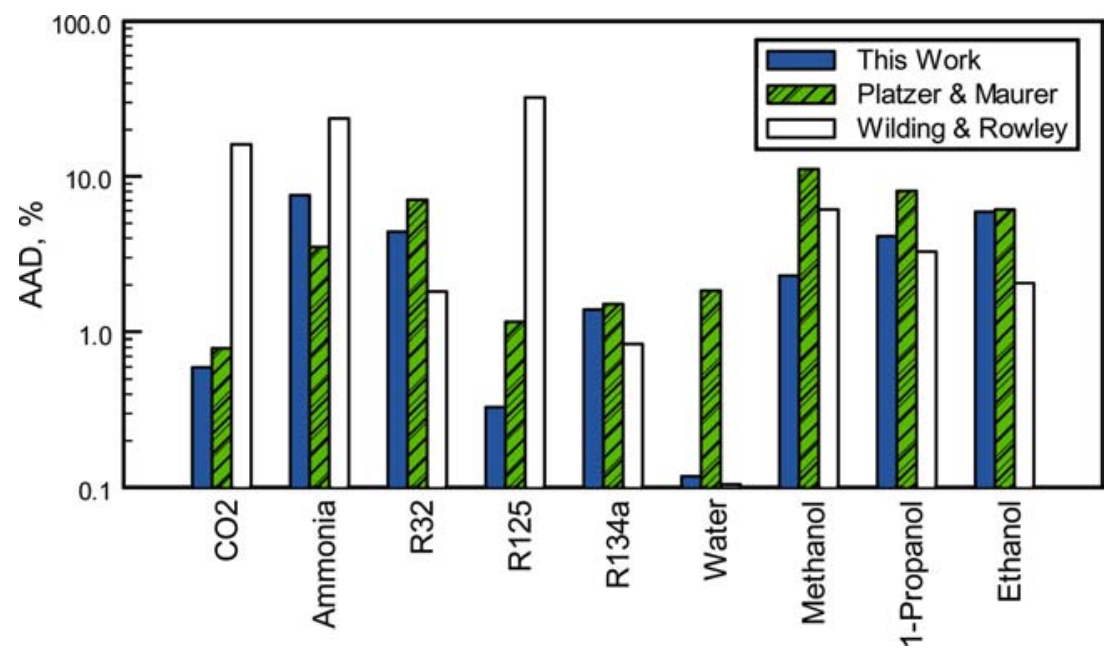

Fig. 8. Comparisons of average absolute deviations for saturated liquid density calculated from the CSP model and the equations by Platzer and Maurer [26] and by Wilding and Rowley [16] for 9 polar and associating fluids.

As seen in this section, the results for caloric properties for polar and associating fluids are not as good as to those for nonpolar and weakly polar fluids. The generalized technical EOS does, however, give reasonable predictions for the saturation-boundary properties and single-phase densities for all 22 nonpolar and weakly polar, polar, and associating fluids considered in this study.

\section{CONCLUSION}

Accurate thermodynamic properties are widely needed in engineering design and analysis. One of the major and reliable sources of accurate thermodynamic properties is the equation of state. Since high quality experimental data are not readily available for most fluids of industrial interest, even moderately accurate equations are lacking and predictive approaches such as the corresponding states principle are good alternatives.

In this work, we developed a four-parameter CSP model to generalize the universal technical EOS, based on available information on reference fluids such as propane, $n$-octane, and water. This model takes four parameters as independent variables, namely, the critical density and temperature, acentric factor, and a polarity factor. When applied to 22 fluids considered in this work, the CSP model shows good accuracy and offers 
the flexibility to be extended to other fluids, compared to other generalized equations such as the one by Span and Wagner for nonpolar fluids [27], and those by Platzer and Maurer [26] and by Wilding and Rowley [16] for polar fluids. In addition, we have successfully developed a correlation model for the polarity factor $\beta$ by using QSAR molecular descriptors. This model enables us to estimate the polarity factor from molecular level information of a given fluid and eliminates the need of fitting experimental data.

\section{ACKNOWLEDGMENTS}

The authors are grateful to the Department of Energy, Office of Basic Energy Sciences for financial support under Grant No. DE-FG0395ER14568. The authors would like to thank Dr. Eric W. Lemmon at the National Institute of Standard and Technology for his valuable comments.

\section{LIST OF SYMBOLS}

$a_{m} \quad$ Coefficients of the technical equation of state

A Helmholtz free energy per mole

$i \quad$ Exponents of equation of state

$j \quad$ Exponents of equation of state

$k \quad$ Exponents of equation of state

$M \quad$ Molecular weight, length of equation of state

$P \quad$ Pressure

$R \quad$ Molar gas constant

$T \quad$ Temperature

$t \quad$ Dimensionless temperature

V Volume

z Compressibility

\section{GREEK LETTERS}

$\alpha \quad$ Size/shape factor

$\beta \quad$ Polarity parameter

$\delta \quad$ Dimensionless density

$\gamma \quad$ Critical exponent, exponent of equation of state

$\varepsilon \quad$ Exponent of equation of state

$\tau \quad$ Dimensionless temperature difference

$\rho \quad$ Density

$\omega \quad$ Acentric factor

$\Phi \quad$ Dimensionless Helmholtz free energy 


\section{SUPERSCRIPTS}

$\begin{array}{ll}0,1,2, & \text { Reference fluids } \\ \text { id } & \text { Ideal gas part } \\ r & \text { Residual part }\end{array}$

\section{SUBSCRIPTS}

$\begin{array}{ll}c & \text { Critical } \\ r & \text { Reducing parameters }\end{array}$

\section{REFERENCES}

1. O. Redlich and J. N. S. Kwong, Chem. Rev. 44:233 (1949).

2. D.-Y. Peng and D. B. Robinson, Ind. Eng. Chem. Fund. 15:59 (1976).

3. G. S. Soave, Chem. Eng. Sci. 27:1197 (1972).

4. H. Kamerlingh Onnes, Commun. Phys. Lab., Leiden 71 (1901).

5. E. Bender, presented at the 5th Symp. Thermophys. Prop., New York (unpublished, 1970).

6. W. G. Chapman, K. E. Gubbins, G. Jackson, and M. Radosz, Ind. Eng. Chem. Res. 29: 1709 (1990).

7. S. H. Huang and M. Radosz, Ind. Eng. Chem. Res. 29:2284 (1990).

8. M. Benedict, G. B. Webb, and L. C. Rubin, J. Chem. Phys. 8:334 (1940).

9. R. T. Jacobsen and R. B. Stewart, J. Phys. Chem. Ref. Data 2:757 (1973).

10. W. Wagner and A. Pruss, J. Phys. Chem. Ref. Data 31:387 (2002).

11. R. Span and W. Wagner, J. Phys. Chem. Ref. Data 25:1509 (1996).

12. R. Span, E. W. Lemmon, R. T. Jacobsen, W. Wagner, and A. Yokozeki, J. Phys. Chem. Ref. Data 29:1361 (2000).

13. C. Tegeler, R. Span, and W. Wagner, J. Phys. Chem. Ref. Data 28:779 (1999).

14. K. S. Pitzer, J. Chem. Phys. 7:583 (1939).

15. B. I. Lee and M. G. Kesler, AIChE J. 21:510 (1975).

16. W. V. Wilding and R. L. Rowley, Int. J. Thermophys. 7:525 (1986).

17. B. Saager, R. Hennenberg, and J. Fischer, Fluid Phase Equilib. 72:41 (1992).

18. J. F. Ely, Adv. Cryog. Eng. 35:1511 (1990).

19. J. Gmehling, K. Fischer, J. Li, and M. Schiller, Pure Appl. Chem. 55:919 (1993).

20. P. G. Toledo and R. Reich, Ind. Eng. Chem. Res. 27:1004 (1988).

21. M. A. R. Sharif and T. K. Groves, Chem. Eng. Com. 98:89 (1990).

22. Y. L. Wong, S. C. Cheng, and D. C. Groeneveld, Heat Transfer Eng. 11:60 (1990).

23. S. Ye, B. Lagourette, J. Alliez, H. Saint-Guirons, and F. Montel, Fluid Phase Equilib. 74:157 (1992).

24. D. Garipis and M. Stamatoudis, AIChE J. 38:302 (1992).

25. A. Karkaris, T. Kalfopoulus, and M. Stamatoudis, Ind. Eng. Chem. Res. 31:1830 (1992).

26. B. Platzer and G. Maurer, Fluid Phase Equilib. 51:223 (1989).

27. R. Span, Multiparameter Equations of State - An Accurate Source of Thermodynamic Property Data. (Springer-Verlag, Berlin, 2000).

28. R. Span and W. Wagner, Int. J. Thermophys. 24:1 (2003). 
29. L. Sun and J. F. Ely, Fluid Phase Equilib. 222/223:107 (2004).

30. J. M. Prausnitz, R. N. Lichtenthaler, and E. G. de Azevedo, Molecular Thermodynamics of Fluid-Phase Equilibria (Prentice Hall PTR, Upper Saddle River, New Jersey, 1999).

31. J. F. Ely and I. M. F. Marrucho, in Equations of State for Fluids and Fluid Mixtures, J. V. Sengers, R.F. Kayser, C. J. Peters, and H. J. White, Jr., eds. (Elsevier, Amsterdam, New York, 2000).

32. A. S. Teja and P. Rice, Chem. Eng. Sci. 36:1 (1981).

33. A. S. Teja, S. I. Sandler, and N. C. Patel, Chem. Eng. J. 21:21 (1981).

34. J. H. Keenan, F. G. Keyes, P. G. Hill, and J. G. Moore, Steam Tables (Wiley, New York, 1969).

35. Accelrys, Cerius ${ }^{2}$ V 4.0 (1999).

36. Accelrys, Cerius $^{2}-Q S A R+(2000)$. 